\title{
Coping Strategies as Predictors of Anxiety: Exploring Positive Experience of Chinese University in Health Education in COVID-19 Pandemic
}

\author{
Yue Li1,2, Jun Peng2* \\ ${ }^{1}$ School of Art, Shenzhen University, Shenzhen, China \\ ${ }^{2}$ School of Education, City University of Macau, Macau, China \\ Email: ^junpeng@cityu.mo
}

How to cite this paper: Li, Y., \& Peng, J. (2020). Coping Strategies as Predictors of Anxiety: Exploring Positive Experience of Chinese University in Health Education in COVID-19 Pandemic. Creative Education, 11, 735-750.

https://doi.org/10.4236/ce.2020.115053

Received: April 20, 2020

Accepted: May 12, 2020

Published: May 15, 2020

Copyright () 2020 by author(s) and Scientific Research Publishing Inc. This work is licensed under the Creative Commons Attribution International License (CC BY 4.0).

http://creativecommons.org/licenses/by/4.0/

\begin{abstract}
Background: It is urgent to identify the effective measures to cope with the psychological stress triggered by the precipitate coronavirus disease 2019 (COVID-19) pandemic. Objective: The present study aimed at examining the levels and links of the anxiety and coping strategies, and then exploring the coping predictors in anxiety in the crisis. Methods: An online survey was conducted using stratified random sampling method among 2640 Chinese university students from 21 February to 24 February 2020. The students' anxiety and coping strategies were measured by the Self-Rating Anxiety Scale (SAS) and the Coping Strategy Questionnaire (CSQ), respectively. Results: The students expressed high levels of coping strategies and comparatively low level of anxiety. Multiple stepwise regression analysis showed that cognitive coping, emotional coping, and social support had a significantly negative predictive effect on anxiety, social support being the most powerful predictor, whereas behavioral coping predicted anxiety positively. Conclusion: The findings suggest that the enhancement of cognitive coping, emotional coping, and social support should be highlighted and the potentially destructive impact of behavioral coping is noteworthy, and the pedagogical implications in health education are hereby addressed.
\end{abstract}

\section{Keywords}

Coping Strategy, Anxiety, Predictor, Experience,

Chinese University Students, COVID-19 Pandemic,

Health Education 


\section{Introduction}

The prolonged threat of the coronavirus disease 2019 (COVID-19) pandemic has swept the globe since the declaration of the World Health Organization (WHO) on 31 December 2019 (World Health Organization, 2020). Although most countries have taken great effort to implement emergent countermeasures to restrain the aggressive escalation of the pandemic, the ongoing numbers of infection have never decelerated for a while. The pandemic is so unprecedented that it has gone through 210 countries and territories, with more than two million cases and one hundred and thirty thousand deaths, as of April 16, 2020 (Worldometer, 2020).

The infectious disease has not only affected the physical health of the patients, but also the psychological wellbeing of the uninfected population. Prior studies have revealed a high prevalence of posttraumatic stress and depressive symptoms associated with long quarantine (Hawryluck et al., 2004). A recent study of 1210 Chinese public has also reported that $53.8 \%$ of respondents perceived moderate or severe psychological impact during the initial outbreak of the COVID-19 pandemic (Wang et al., 2020). Accordingly, these mental disorders are reflected in inappropriate actions as well. The commons have attempted to take various exaggerated coping strategies. Take stock piling for instance. People rushed to the pharmacies or supermarkets to stock surgical masks and groceries far beyond what they needed, which emptied the supplies of many stores (Ho, Chee, \& Ho, 2020; Hsu, Chia, \& Vasoo, 2020). Except for these overreactions, the misuse of information is also a typical respond to a substantial risk. People tend to surge for the information about virus excessively and the increasing uncertainty triggered by the rumors or false information may drive them even more anxious. In the previous study of influenza A (H1N1) case, people were demanded to be more qualified "Netizen" in selecting and evaluating online sources (Kim, Pinkerton, \& Ganesh, 2012). As a result, prompt and accurate reactions to ease the psychological stress deserve more attention (Ioannidis, 2020).

In contrast to the exponentially increased number of cases in other countries, the dissemination of the pandemic in China has been suspended gradually with 46 new cases and one death, of which 36 were immigrated cases, as of April 14, 2020. This is due to a series of extreme public health measures initiated on 23 January 2020, such as resolute shutdown of the cities, rigorous restriction of all public emigration, mandatory quarantine of the potential cases, monitored tracing of contacted individuals, stepwise extension of the holidays, complete hospitalization of the diagnosed patients, early closure of schools, available assistance of psychological interventions, timely release of health information and so on (Yue et al., 2020). In particular, the Chinese universities have resorted to supporting risk management, consisting of raising medical supplies, carrying out specialized medical research, conducting innovative online teaching, 
and providing psychological assistance, which have astricted the devastating impact on psychology (Wang, Cheng, Yue, \& McAleer, 2020). In view of Chinese remarkable achievements, it is essential to explore Chinese coping experience against the epidemic.

The present study, therefore, aims to examine levels and relationship between the coping strategies and anxiety among Chinese university students, probing into the predictive power of coping strategies in determining the variance of anxiety in the context of COVID-19 pandemic. The findings from this study may provide empirical evidence for the implementation of health educations to promote positive experience in universities during the severe period.

\section{Literature Review}

\subsection{Mental Health in the COVID-19 Pandemic}

The significance of mental health has aroused a great concern of worldwide researchers recently, along with the focus on the physical fitness (Carvalho, Moreira, de Oliveira, Landim, \& Neto, 2020; Lima et al., 2020; Zandifar \& Badrfam, 2020). To be particular, mental health pressure originated from multiple populations, such as older adults, medical staff, and youngsters, have invoked the specific exploration of various studies. Chinese scholars noticed the limited access to internet services and urged sufficient and adequate attention paid to the vulnerable older adults (Yang et al., 2020). Considering the considerable mental burden endured by the medical care workers in the frontline, various approaches of psychological interventions were adopted in the Chinese hospitals, including provision of comforting accommodation, pre-service psychological training, development of protective equipment, propaganda of relaxing measures, and contact of psychological counsellors (Chen et al., 2020). Extended home confinement brought psychosocial stressors to children and adolescents, such as fears of infection, tediousness, annoyance, lack of in-person contact with peers and teachers, of which the Chinese government should raise the awareness (Wang, Zhang, Zhao, Zhang, \& Jiang, 2020). Furthermore, the findings of empirical studies sampled with 1257 Chinese heath care workers demonstrated that women, nurses, and frontline doctors in Wuhan were more affected by depression, anxiety, insomnia, and distress (Lai et al., 2020). The investigation of 1,210 general public portrayed a statistical picture of the psychological responses in China, indicating that one-third of the respondents experienced moderate and severe anxiety (Wang et al., 2020).

\subsection{Coping Strategies Related to Anxiety}

Coping strategies can be regarded as certain cognitive or behavioral respond towards stressors beyond individual capacities (Lazarus \& Folkman, 2013). Abundant studies have elaborated the potential coping strategies associated with anxiety (Bovero et al., 2019; Ciobanu et al., 2020; Wu, Xu, Zhang, \& Liu, 2019). A 
widely accepted classification of coping strategy is positive and negative coping, while the positive one including problem-solving and seeking suggestions seems to mitigate the destructive effect of stress (Vinothkumar et al., 2016). Researchers have offered a variety of proposals regarding mental health strategies to combat the panic and distress during this unusual phase, composed of detecting the sensitive citizens, promoting screening interventions, enhancing social support, and integrating professional resources (Ho et al., 2020). Emergent countermeasures in Sichuan Province, China, were presented to meet the psychological challenge, which introduced the issue of self-assistance manual, operation of 24-hour psychological hotlines, and application of online survey (Zhou, 2020). A narrative literature review provided systematic insights into the coping actions and psychological consequences on the basis of twenty-four published articles targeting at this pandemic (Chew, Wei, Vasoo, Chua, \& Sim, 2020). According to this narration, the appearance of psychological anxiety was prevalent across the previous studies, with rates ranging from $3.2 \%$ to $12.6 \%$, and positive coping was positively associated with perceived health and life satisfaction which in turn alleviated psychological symptoms (Chew et al., 2020). It was also suggested that public should pay more attention to coping strategies (i.e., updating reliable information about the disease, raising awareness of the psychological impact, positive appraisal of the situation, seeking protective alternatives, and empowering social support) which framed the variables of coping strategies (i.e., cognitive coping, emotional coping, behavioral coping, and social support) in the present study. In addition, the demographic factors, such as student status and self-rated health status, have been proved to influence the practical response and anxiety among the Chinese general public (Wang et al., 2020). These findings laid a foundation of the research questions in this study.

\section{Research Questions}

Previous studies have mentioned the significance of coping strategies in reducing anxiety either in narrative or empirical approaches, but rare empirical studies so far have explored the relationship between coping strategies and anxiety in sample of university students. Accordingly, the present study aims to identify the predictors related to coping strategies on anxiety in COVID-19 pandemic based on samples of Chinese university students. More specifically, this study will address the following four research questions (RQs):

RQ1: To what extent do Chinese students adopt coping strategies and suffer from anxiety in the context of COVID-19 pandemic?

RQ2: Do levels of Chinese university students' anxiety and coping strategies vary among population with different sociodemographic characteristic in the context of COVID-19 pandemic?

RQ3: What are the links between the coping strategies (i.e., cognitive coping, emotional coping, behavioral coping, and social support) and participants' anxiety in the context of COVID-19 pandemic? 
RQ4: What are the predictive effects of coping strategies on students' anxiety in the context of COVID-19 pandemic?

\section{Method}

\subsection{Participants}

A total of 2640 university students (1816 females, 824 males) from a comprehensive university in Shenzhen, a demonstration pilot zone for socialism with Chinese characteristics in Southern China, participated in this study. The students investigated were composed of graduate students $(\mathrm{N}=383)$ and undergraduates $(\mathrm{N}=2257)$ which included 677 freshmen, 616 sophomores, 545 juniors, and 588 seniors. A small number of the participants $(\mathrm{N}=62)$, among which 24 students were medically observed, were isolated in Hubei Province, a province with considerably severe pandemic in the early phase of the outbreak, while a great majority $(\mathrm{N}=2578)$ were isolated at home other than Hubei Province. In addition, their current self-efficacy on health was reflected by self-perceived health status, of which $80.91 \%(\mathrm{~N}=2136)$ deemed they were very healthy, $17.61 \%(\mathrm{~N}=465)$ confirmed their healthy condition, $1.4 \%$ were not sure for their current health status, and only 2 student (percentage $=0.08 \%$ ) considered themselves not healthy. As for the psychological conditions, only $0.53 \%(\mathrm{~N}=14)$ expressed the need for psychological interventions while 2,626 students (percentage $=99.47 \%$ ) declined the professional assistance. The above demographic information is summarized in Table 1.

Table 1. Participants' sociodemographic information.

\begin{tabular}{|c|c|c|c|}
\hline Sociodemographic Variables & Categories & Frequency & Percentage \\
\hline \multirow{3}{*}{ Gender } & Male & 824 & 31.21 \\
\hline & & & \\
\hline & Female & 1816 & 68.79 \\
\hline \multirow{2}{*}{ Academic degree } & Graduate student & 383 & 14.51 \\
\hline & Undergraduate & 2257 & 85.49 \\
\hline \multirow{2}{*}{ Current location } & Hubei Province (the epicenter) & 62 & 2.35 \\
\hline & Other province & 2578 & 97.65 \\
\hline \multirow{2}{*}{ Isolation condition } & Medically observed & 24 & 0.91 \\
\hline & Not isolated & 2616 & 99.09 \\
\hline \multirow{4}{*}{ Self-perceived health status } & Very healthy & 2136 & 80.91 \\
\hline & Healthy & 465 & 17.61 \\
\hline & Not sure & 37 & 1.4 \\
\hline & Not healthy & 2 & 0.08 \\
\hline \multirow{2}{*}{ Psychological conditions } & Need for interventions & 14 & 0.53 \\
\hline & No need for interventions & 2626 & 99.47 \\
\hline
\end{tabular}




\subsection{Research Instruments}

The Sociodemographic Characteristic Questionnaire (SCQ), the Coping Strategy Questionnaire (CSQ), and the Self-Rating Anxiety Scale (SAS) were used as self-report instruments.

\section{The Sociodemographic Characteristic Questionnaire (SCQ)}

The investigation started with a demographics section from which the information about participants' gender, academic degree (graduate student or undergraduate), geographical background (Hubei Province, the epicenter or not), isolation background (medically observed or not), self-perceived health background, and psychological conditions was retrieved.

\section{The Coping Strategy Questionnaire (CSQ)}

The coping strategies adopted by university students in the COVID-19 pandemic were measured by the Coping Strategy Questionnaire (CSQ) of which there were four subscales. The former three subscales (i.e., cognitive coping, emotional coping and behavioral coping) were enlightened by Lazarus and Folkman's (2013) definition of coping as the cognitive, emotional, and behavioral respond confronted with stress. The forth subscale (i.e., social support) was revised from the Chinese version of the SSQ to assess the levels of social support received by the university students (Xiao \& Yang, 1987). This fifteen-item scale employed 5-point Likert-type ranging from "strongly disagree" to "strongly agree" (scored 1 - 5) with subscales of cognitive coping (items 1 - 3), emotional coping (items 4 - 6), behavioral coping (items 7 - 10) and social support (items $11-15$ ). The higher the scores were, the higher levels of positive coping strategies the students adopted. The items for each subscale are described in Table 2.

Principal components analyses were conducted to evaluate the validity of this scale (see Table 2). The Kaiser-Meyer-Olkin test was first performed to verify the feasibility of using principal components analysis in this study, yielding an index of 0.866 on the scale. Subsequently, the significant $(p=0.000)$ outcome in the Bartlett's test of sphericity ensured the factor model conducted by the exploratory factor analysis. The results of the factor analysis for the 15 items were specified in Table 2. Four factors emerged which accounted for a total of $65.44 \%$ of the variance eventually. Factor 1 consisted of the first three items, representing cognitive coping (CC) with the factor coefficients all higher than 0.6. Factor 2, which involved the following three items (factor coefficients $>0.7$ ), represented emotional coping (EC). The factor coefficients of items in Factor 3 which signified behavioral coping (BC) were totally taller than 0.6. Factor 4 labeled as social support (SS) were composed of four items (item 11 - 15), with all factor coefficients larger than 0.6. Consequently, the irrelevance of the resulting variables (CC, EC, BC, SS) generated from the exploratory factor analysis could be testified, which reinforced the validity of the follow-up analyses. Moreover, the internal consistency reliability was accessed by the values of the Cronbach's alpha coefficients. The Cronbach's alpha coefficient of the whole scale was 0.83 , with $0.70,0.67,0.77$, and 0.89 for each subscale dimension of the cognitive coping, emotional coping, behavioral coping, and social support, respectively. 
Table 2. Exploratory factor analysis.

\begin{tabular}{clll}
\hline \multicolumn{1}{c}{ Items } & FC & FL \\
\hline 1 & I am aware of the preventive information about the COVID-19. & 0.822 & \\
2 & I have paid close attention to the news about the epidemic. & 0.74 & CC \\
3 & I suppose that the epidemic should be under effective control. & 0.639 & \\
\hline 4 & I am worried about being infected by the COVID-19. & 0.734 & EC \\
5 & I am afraid that everyone could be the infected patient. & 0.813 & \\
6 & I fell unsafe even if I wear mask outside. & 0.771 & \\
\hline 7 & I wear mask when I go outside. & 0.751 & \\
8 & I have paid more attention to the personal health than before. & 0.717 & BC \\
9 & I have cancelled the schedule of outing and social gathering. & 0.777 & \\
10 & I have tried to avoid going to hospital in case of infection. & 0.635 & \\
\hline 11 & I made self-examination in face of metal stress in the epidemic. & 0.651 & \\
12 & I talk to my friends in face of metal stress in the epidemic. & 0.751 & \\
13 & I talk to my families in face of metal stress in the epidemic. & 0.661 & SS \\
14 & I talk to my instructors in face of metal stress in the epidemic. & 0.909 & 0.911 \\
15 & I talk to my counsellors in face of metal stress in the epidemic. & & \\
\hline
\end{tabular}

Note: $\mathrm{FC}=$ Factor Coefficient, $\mathrm{FL}=$ Factor Label, $\mathrm{CC}=$ Cognitive Coping, $\mathrm{EC}=$ Emotional Coping, $\mathrm{BC}=$ Behavioral Coping, SS = Social Support.

\section{The Self-Rating Anxiety Scale (SAS)}

The levels of anxiety among university students were obtained through the Self-Rating Anxiety Scale (SAS) (Olatunji, Deacon, Abramowitz, \& Tolin, 2006). The rough scores of the SAS questionnaire which consisted of 20 four-point-scale items were then multiplied by 1.25 to generate the aggregated scores, with higher scores directing more severe levels of anxiety. The Cronbach's alpha coefficient for the SAS was 0.89 in this research, which indicated acceptable reliability of this scale.

\subsection{Data Collection}

The research ethical approval of investigation in coping status towards metal stress in the COVID-19 epidemic was granted by the first author's institution. Given the unavailability to conduct the survey in a traditional paper-and-pencil way during the special period, the anonymous questionnaires were completed via a web survey, with stratified random sampling in terms of students' gender, major, and academic degree. All the 2640 participants were informed of the purpose, necessity and the demand for the investigation before they filled in the questionnaires by means of the introduction about the survey provided on the first page of the questionnaires. The data were collected during February 21st and 24th of 2020 when the students had been isolated at home for more than one month since the outbreak of the COVID-19 epidemic in China. 


\subsection{Data Analysis}

The data were processed by means of IBM SPSS statistics 24.0 for Windows (IBM Corp., Armonk, NY, USA). To address the first research question, descriptive statistics were used to analyze the means and the standard deviation of the investigated variables. To answer the second research question, one-sample Kolmogorov-Smirnov test was first employed to test the normality of data distribution and then nonparametric analyses were performed to compare the difference in the students' levels of cognitive coping, emotional coping, behavioral coping, social support, and anxiety among population groups with distinct sociodemographic characteristics. For the third research question, Spearman's correlation analyses were used to explore the relationship between coping strategies and anxiety. Furthermore, multiple regression analysis was run to examine the predictive power of the independent variables (i.e., coping strategies and their subscales) for variance of anxiety.

\section{Results}

\subsection{The Levels of Coping Strategies and Anxiety}

It was found in the descriptive data summarized in Table 3 that the level of students' total coping strategy was high $(\mathrm{M}=4.26, \mathrm{SD}=0.35)$, among which behavioral coping showed the highest level $(\mathrm{M}=4.76, \mathrm{SD}=0.37)$ and emotional coping remained the lowest level $(\mathrm{M}=2.76, \mathrm{SD}=0.87)$. Meanwhile, in contrast to the Chinese norm of the SAS ( $\mathrm{M}=29.78, \mathrm{SD}=0.46)$ during the normal time, the students experienced relatively higher anxiety during the epidemic period $(\mathrm{M}=36.25, \mathrm{SD}=4.57)(\mathrm{Li}$ et al., 2004).

\subsection{The Difference in Coping Strategies and Anxiety among Sociodemographic Variables}

One-sample Kolmogorov-Smirnov tests revealed that the distribution of anxiety was not normal (KS $=0.204, p<0.001)$. Accordingly, Mann-Whitney $\mathrm{U}$ tests and Kruskal-Wallis $\mathrm{H}$ tests were conducted to identify the difference in coping strategies and anxiety among sociodemographic variables (i.e., gender, academic degree, geographical background, isolation condition, and self-perceived health status). Non-parametric analyses showed that there were no significant difference in coping strategies and anxiety among students with various gender, academic degree and geographical background $(p>0.05)$. However, students with distinct self-perceived health status differed significantly in terms of total coping strategies $(p<0.01)$, emotional coping $(p<0.05)$, behavioral coping $(p<0.01)$, social support $(p<0.01)$, and anxiety $(p<0.01)$. To be more specific, students who considered themselves healthier adopted more positive coping strategies, particularly in emotional coping, behavioral coping, and social support, and suffered less anxiety than the counterparts with low self-perceived health status. Moreover, it was worthy of note that the students medically observed possessed significantly lower emotional coping than the non-isolated students $(p<0.05)$ (see Table 4). 
Table 3. The levels of coping strategies and anxiety in the pandemic.

\begin{tabular}{cccccccc}
\hline \multirow{2}{*}{ Descriptive } & \multicolumn{5}{c}{ CS } & \multirow{2}{*}{ A } \\
\cline { 2 - 6 } & TCS & CC & EC & BC & SS & \\
\hline M & 4.26 & 4.53 & 2.76 & 4.76 & 4.56 & 36.25 \\
SD & 0.35 & 0.47 & 0.87 & 0.37 & 0.58 & 4.57 \\
\hline
\end{tabular}

Note: $\mathrm{TCS}=$ Total Coping Strategies, $\mathrm{CC}=$ Cognitive Coping, $\mathrm{EC}=$ Emotional Coping, $\mathrm{BC}=$ Behavioral Coping, $\mathrm{SS}=$ Social Support, $\mathrm{A}=$ Anxiety, $\mathrm{M}=$ Mean, $\mathrm{SD}=$ Standard Deviation.

Table 4. Difference in coping strategies and anxiety among variables of isolation condition and self-perceived health status.

\begin{tabular}{ccccccc}
\hline \multirow{2}{*}{ Variables } & TCS & CC & EC & BC & SS & A \\
\cline { 2 - 7 } & \multicolumn{7}{c}{ Median } \\
\hline Isolation condition & & & & \\
Medically observed & 4.25 & 4.83 & 2.17 & 5 & 4.9 & 36 \\
Not isolated & 4.31 & 4.67 & 2.67 & 5 & 5 & 35 \\
Mann Whitney U value & 0.84 & -1.63 & 2.47 & -1.25 & 0.23 & -0.66 \\
$p$ & 0.402 & 0.103 & $0.013^{*}$ & 0.21 & 0.822 & 0.512 \\
Self-perceived health status & & & & & & \\
Very healthy & 4.38 & 4.67 & 2.67 & 5 & 5 & 35 \\
Healthy & 4.06 & 4.33 & 2.67 & 4.75 & 4 & 38 \\
Not sure & 3.81 & 4 & 2.67 & 4.5 & 4 & 39 \\
Not healthy & 3.25 & 4.33 & 2 & 4.25 & 2.6 & 45 \\
Kruskal-Wallis H value & 303.71 & -1520.22 & 11.09 & 125.97 & 103.52 & 214.66 \\
$p$ & $0.000^{* *}$ & 1 & $0.011^{*}$ & $0.000^{* *}$ & $0.000^{* *}$ & $0.000^{* *}$ \\
\hline
\end{tabular}

Note: ${ }^{*} p<0.05,{ }^{* *} p<0.01$; TCS $=$ Total Coping Strategies, CC = Cognitive Coping, $\mathrm{EC}=$ Emotional Coping, $\mathrm{BC}=$ Behavioral Coping, $\mathrm{SS}=$ Social Support, $\mathrm{A}=$ Anxiety.

\subsection{The Links between Coping Strategies and Anxiety}

The Spearman correlations between coping strategies and anxiety are shown in Table 5. The results revealed that anxiety was significantly and negatively associated with total coping strategies $(\mathrm{r}=-0.287, p<0.01)$ and their subscales. More specifically, the correlation coefficient between anxiety and social support $(\mathrm{r}=-0.293, p<0.01)$ was the highest while that between anxiety and emotional coping $(\mathrm{r}=-0.135, p<0.01)$ was the lowest. The finding that all independent variables were significantly correlated laid the room for the regression analysis.

\subsection{The Coping Predictors of Anxiety}

A multiple stepwise regression model of anxiety was estimated, using the four subscales of coping strategies as independent variables (see Table 6). The overall model fit statistics were $\mathrm{F}=99.005, p=0.000$ with adjusted $\mathrm{R}^{2}=0.129$. The $\mathrm{F}$ statistics showed rejection of the null hypothesis of insignificance for all independent variables, and the variance inflation factors (VIF) of all the predictor variables were lower than 5 , revealing no danger of multicollinearity in the model. 
Table 5. Inter-correlations between coping strategies and anxiety.

\begin{tabular}{ccccccc}
\hline Variables & A & TCS & CC & EC & BC & SS \\
\hline A & 1 & & & & & \\
TCS & $-0.287^{* *}$ & 1 & & & & \\
CC & $-0.218^{* *}$ & $0.650^{* *}$ & 1 & & & \\
EC & $-0.135^{* *}$ & $0.433^{* *}$ & -0.021 & 1 & & \\
BC & $-0.141^{* *}$ & $0.613^{* *}$ & $0.462^{* *}$ & $-0.097^{* *}$ & 1 & 1 \\
SS & $-0.293^{* *}$ & $0.788^{* *}$ & $0.526^{* *}$ & -0.024 & $0.564^{* *}$ & 1 \\
\hline
\end{tabular}

Note: ${ }^{*} p<0.01 ; \mathrm{A}=$ Anxiety, $\mathrm{TCS}=$ Total Coping Strategies, $\mathrm{CC}=$ Cognitive Coping, $\mathrm{EC}=$ Emotional Coping, BC $=$ Behavioral Coping, $\mathrm{SS}=$ Social Support.

Table 6. Multiple regression analysis of anxiety on the subscales of coping strategies.

\begin{tabular}{cccccccc}
\hline Variables & $\mathrm{B}$ & $\mathrm{SE}$ & $\mathrm{t}$ & $p$ & $\mathrm{VIF}$ & $R^{2}$ & Adjusted $R^{2}$ \\
\hline (constant) & 48.42 & 1.17 & 41.39 & $0.000^{* *}$ & - & & \\
Social support & -2.63 & 0.18 & -14.73 & $0.000^{* *}$ & 1.59 & & \\
Behavioral coping & 0.99 & 0.28 & 3.57 & $0.000^{* *}$ & 1.56 & 0.131 & 0.129 \\
Emotional coping & -0.77 & 0.10 & -8.05 & $0.000^{* *}$ & 1.01 & \\
Cognitive coping & -0.58 & 0.21 & -2.73 & $0.006^{* *}$ & 1.43 & \\
& \multicolumn{7}{c}{$\mathrm{F}=99.005, p=0.000$} \\
\end{tabular}

Note: ${ }^{* *} p<0.01$.

The qualification of the multicollinearity test verified the significance of the regression equation model (Tabachnick \& Fidell, 2001). Generally, the coping strategy model explained $13.1 \%$ of the variance in anxiety. All the following facets of coping strategies were significant predictors of anxiety, which were ranked in order from strongest to weakest according to the beta weights: social support ( -2.63$)$, behavioral coping (0.99), emotional coping ( -0.77$)$, and cognitive coping $(-0.58)$. The variables of social support, emotional coping, and cognitive coping predicted anxiety negatively, indicating that students with higher levels of social support, emotional coping, and cognitive coping showed less anxiety. Surprisingly, the estimated regression coefficient of the behavioral coping variable was positive which indicated that students who had more positive behaviors against the pandemic reported a higher level of anxiety.

\section{Discussion}

The present study examined the coping strategies and anxiety which Chinese university students had gone through during the COVID-19 pandemic. In particular, we were interested in exploring the potential coping predictors of anxiety. To the best of our knowledge, this is the first empirical research to investigate the relationship between coping strategies and anxiety among Chinese university students in the context of COVID-19 epidemic. 
The first research question explored the levels of the coping strategies and anxiety among Chinese students in the COVID-19 pandemic. Participants reported a high level of total coping strategies, particularly in behavioral coping, social support, and cognitive coping. These findings suggests that students are fully aware of the significance of the pandemic and obey the health guidance strictly, refraining from attending social gathering. These findings are supported by a previous study that a majority of the Chinese general population acquired knowledge about COVID-19 via various multimedia, and $75.1 \%$ of the respondents were satisfied with the health information available (Wang et al., 2020). Meanwhile, they adopted precautionary measures more frequently than before, and the statistics that $75.2 \%$ of respondents concerned about their family members getting infected demonstrated a firm family support among Chinese families (Wang et al., 2020). The finding of low emotional coping is universal that the investigation in China and America all confirmed. The study sampled with Chinese online Weibo users reported a climbing detrimental emotions and sensitivity to social risks since the declaration of COVID-19 pandemic (Li, Wang, Xue, Zhao, \& Zhu, 2020). Similarly, it was found in the poll of 2200 American conducted by the Morning Consult Company from January 24-26, 2020, when only 5 cases of infection occurred that over a third of participants (37\%) showed more worries for the dissemination of the coronavirus (Asmundson \& Taylor, 2020). Compared with the Chinese norm of the SAS $(M=29.78, S D=0.46)$ during the normal time, the students experienced relatively higher anxiety $(\mathrm{M}=$ 36.25 , SD $=4.57$, which is however much lower than the self-isolated population $(\mathrm{M}=55.38, \mathrm{SD}=14.29)$ in central China during the epidemic period (Xiao, Zhang, Kong, Li, \& Yang, 2020b).

The second research question focused on the difference in coping strategies and anxiety among sociodemographic variables. No gender difference was found in participant's anxiety, which is inconsistent with the Chinese finding that male gender showed significant higher anxiety than female in the pandemic, whereas the finding of academic background is similar to that study where educational attainment was not associated with anxiety scores (Wang et al., 2020). In contrast to the finding in the above study that recent quarantine in the past 14 days exerted no effect on the respond to the pandemic and the emotional condition, the present study found that students who were medically observed employed more negative coping emotionally. In other words, the countermeasures of isolation, quarantine, social distancing and community containment generated great negative psychological consequences to the individuals (Wilder-Smith \& Freedman, 2020). One possible explanation is that the fear of being infected and stress resulting from limited social contact aggravated the metal disorder. The finding that students with various self-perceived health status revealed significant difference in coping strategies and anxiety is in line with the Chinese study which stated a close association with current self-rated health status and psychological impact (Wang et al., 2020).

The third research question examined the links between coping strategies and 
anxiety. Anxiety was significantly and negatively correlated with total coping strategies and their subscales. Students with more positive coping strategies tended to experience less anxiety in the context of COVID-19 pandemic. The negative association between cognitive coping and anxiety can be supported by the previous finding that individuals who kept close contact with the updating accurate health information suffered from lower levels of anxiety (Wang et al., 2020). Previous study also confirmed the constructive function of social support on mental health in that children with less family members, peer support, and school friends in impoverished families were prone to endure more miserable mental distress ( $\mathrm{Li}$, Jiang, \& Fang, 2017). Positive coping strategies, such as problem solving or looking for advice, are supposed to diminish stress while negative ones, including self-blame, avoidance, wishful thinking, are likely to reinforce the extent of stress (Vinothkumar et al., 2016).

The fourth research question dealt with the predictive power of all the coping strategies on the Chinese university students' anxiety during the COVID-19 pandemic. Multiple regression analyses revealed that cognitive coping, behavioral coping, emotional coping, and social support predicted $13.1 \%$ of variance in anxiety. To be more specific, the negative regression coefficients of cognitive coping, emotional coping and social support with anxiety indicated that students with more positive cognitive coping, emotional coping, and social support tended to experience lower anxiety during the pandemic. It is noticeable that social support was the most powerful predictor among the coping predictors of anxiety. The facilitating effect of social support has been verified in the prior study that social support of the medical staff who treated the infected patients could reduce anxiety by means of sharing empathy among families and friends (Xiao, Zhang, Kong, Li, \& Yang, 2020a). Out of our expectations, behavioral coping tuned out to be a positive predictor on anxiety, which is inconsistent with the relevant finding that individuals with particular precautionary measures, such as hand hygiene and wearing a mask, obtained less psychological distress (Wang et al., 2020). It can be inferred that students who engaged in excessive protective reaction, such as washing hands much more frequently than needed and wearing multilayer masks were inclined to be more anxious.

Given the comparatively low level of anxiety and high levels of coping strategies among Chinese university students, it is absolutely imperative to explore the positive experience in health education under the circumstance of the COVID-19 pandemic. To begin with, regarding the coping predictors, the most predictive power of social support should be emphasized. To be more specific, universities should be encouraged to provide professional support, such as constructing self-support and family support groups by means of counselling guidance. A majority of Chinese universities have provided consistent psychological assistance since the outbreak of the pandemic. Take Tsinghua University in Beijing for instance. An online emergency public welfare project named as "Combating Epidemics and Psychological Assistance" was launched to improve the mental health of medical staff in the frontline and the public (Wang, Cheng, Yue, \& 
McAleer, 2020). Shanxi Normal University published the first Mental Health Guidance Manual in China as well, with the purpose of sustaining the physical and mental health of the public (Wang et al., 2020). Second, considering the positive predictive effect of emotional coping, educators and administrators in universities should advocate the confidence in professional authorities and government administration to optimize the students' emotional respond (Yue et al., 2020). Meanwhile, students and the public should be instructed to avail of the online platform or counselling hotlines in case of psychological interventions. Counselling training centers, student affairs offices, and student health centers should be integrated for the release of health information, guidance of physical hygiene, and concern of mental status to ease off the students' psychological stress (Zhai \& Du, 2020). Third, the positive predictive impact of cognitive coping should also be highlighted, and students should be informed of the updated and verifiable information. This can be embodied in the appropriate comprehension of health information as a result that rumors and exaggerated information may lead to overreaction, anxiety, and depression. Fourth, with regard to the potential negative effect of behavioral coping, public should be alarmed against excessive behavioral coping to maintain stable mental health. Timely and objective information about the risk of transmission and protective measures should be involved in public health education (Xiao \& Torok, 2020).

The present study has some limitations. Firstly, all the variables in the study were self-reported that may not completely reflect the actual levels of anxiety and coping strategies. However, the questionnaires were conducted in an anonymous way to avoid social bias. Secondly, the data were collected from one university, limiting the generalization of our findings. Thirdly, alternate methodologies apart from cross-sectional study, such as longitudinal or experimental designs, should be employed to verify the findings of this study.

\section{Conclusion}

The current results entail the first preliminary empirical evidence that positive coping strategies have predictive power upon anxiety in the context of COVID-19 pandemic. In general, high coping strategies and comparatively low anxiety existed among the Chinese university students at the early phase of the pandemic. Students' isolation condition and self-perceived health status had significant effect on coping strategies and anxiety while gender and academic attainment had no effect. Multiple regression analysis showed that the four coping variables (i.e., cognitive coping, emotional coping, behavioral coping, and social support) were significant predictors of anxiety in the epidemic circumstance.

In summary, this research contributes to expanding the understanding of the psychological implications of anxiety by suggesting the potential coping predictors in the COVID-19 pandemic. It is of great value to take advantage of effective coping strategies to conquer the negative mental consequences as soon as possible. The findings of this study are expected to provide positive experience for health education and administration in universities. 


\section{Acknowledgements}

The authors thank all the participants who took part in the research. This study was funded by the key project of Physical and Psychological Health and Artistic Expression Studio, Shenzhen University (Grant number 19XSZ03).

\section{Conflicts of Interest}

The authors declare no conflicts of interest regarding the publication of this paper.

\section{References}

Asmundson, G. J. G., \& Taylor, S. (2020). Coronaphobia: Fear and the 2019-nCoV Outbreak. Journal of Anxiety Disorders, 70, Article ID: 102196. https://doi.org/10.1016/j.janxdis.2020.102196

Bovero, A., Tosi, C., Botto, R., Opezzo, M., Giono-Calvetto, F., \& Torta, R. (2019). The Spirituality in End-of-Life Cancer Patients, in Relation to Anxiety, Depression, Coping Strategies and the Daily Spiritual Experiences: A Cross-Sectional Study. Journal of Religion \& Health, 58, 2144-2160. https://doi.org/10.1007/s10943-019-00849-z

Carvalho, P. M. M., Moreira, M. M., de Oliveira, M. N. A., Landim, J. M. M., \& Neto, M. L. R. (2020). The Psychiatric Impact of the Novel Coronavirus Outbreak. Psychiatry Research, 286, Article ID: 112902. https://doi.org/10.1016/j.psychres.2020.112902

Chen, Q., Liang, M., Li, Y., Guo, J., Fei, D., Wang, L., \& Zhang, Z. (2020). Mental Health Care for Medical Staff in China during the COVID-19 Outbreak. The Lancet Psychiatry, 7, e15-e16. https://doi.org/10.1016/S2215-0366(20)30078-X

Chew, Q. H., Wei, K. C., Vasoo, S., Chua, H. C., \& Sim, K. (2020). Narrative Synthesis of Psychological and Coping Responses towards Emerging Infectious Disease Outbreaks in the General Population: Practical Considerations for the COVID-19 Pandemic. Sin gapore Medical Journal, 1-13. https://doi.org/10.11622/smedj.2020046

Ciobanu, I., Di Patrizio, P., Baumann, C., Schwan, R., Vlamynck, G., Bedes, A. et al. (2020). Relationships between Coping, Anxiety, Depression and Health-Related Quality of Life in Outpatients with Substance Use Disorders: Results of the SUBUSQOL Study. Psychology Health \& Medicine, 25, 179-189. https://doi.org/10.1080/13548506.2019.1679847

Hawryluck, L., Gold, W. L., Robinson, S., Pogorski, S., Galea, S., \& Styra, R. (2004). SARS Control and Psychological Effects of Quarantine, Toronto, Canada. Emerging Infectious Diseases, 10, 1206-1212. https://doi.org/10.3201/eid1007.030703

Ho, C. S., Chee, C. Y., \& Ho, R. C. (2020). Mental Health Strategies to Combat the Psychological Impact of COVID-19 beyond Paranoia and Panic. Annals of the Academy of Medicine, Singapore, 49, 1-3.

Hsu, L. Y., Chia, P. Y., \& Vasoo, S. (2020). A Midpoint Perspective on the COVID-19 Pandemic. Singapore Medical Journal, 1-10. https://doi.org/10.11622/smedj.2020036

Ioannidis, J. P. A. (2020). Coronavirus Disease 2019: The Harms of Exaggerated Information and Non-Evidence-Based Measures. European Journal of Clinical Investigation, 50, e13223. https://doi.org/10.1111/eci.13222

Kim, S., Pinkerton, T., \& Ganesh, N. (2012). Assessment of H1N1 Questions and Answers Posted on the Web. American Journal of Infection Control, 40, 211-217. https://doi.org/10.1016/j.ajic.2011.03.028 
Lai, J., Ma, S., Wang, Y., Cai, Z., Hu, J., Wei, N. et al. (2020). Factors Associated with Mental Health Outcomes Among Health Care Workers Exposed to Coronavirus Disease 2019. JAMA Network Open, 3, e203976.

https://doi.org/10.1001/jamanetworkopen.2020.3976

Lazarus, R. S., \& Folkman, S. (2013). Stress: Appraisal, and Coping. New York: Springer.

Li, C., Jiang, S., \& Fang, X. (2017). Effects of Multi-Dimensional Social Capital on Mental Health of Children in Poverty: An Empirical Study in Mainland China. Journal of Health Psychology, 25, 853-867. https://doi.org/10.1177/1359105317737608

Li, S., Wang, Y., Xue, J., Zhao, N., \& Zhu, T. (2020). The Impact of COVID-19 Epidemic Declaration on Psychological Consequences: A Study on Active Weibo Users. International Journal of Environmental Research and Public Health, 17, 2032. https://doi.org/10.3390/ijerph17062032

Li, X. L., Li, X. L., \& Zhang, L. (2004). Stress of SARS and Psychological Health: An Investigation of Response to SARS Stress and Anxiety Level of Nurse Students in SARS Prevalence Period. West China Medical Journal, 19, 596-598.

Lima, C. K. T., Carvalho, P. M. M., Lima, I., Nunes, J., Saraiva, J. S., de Souza, R. et al. (2020). The Emotional Impact of Coronavirus 2019-nCoV (New Coronavirus Disease). Psychiatry Research, 287, Article ID: 112915. https://doi.org/10.1016/j.psychres.2020.112915

Olatunji, B. O., Deacon, B. J., Abramowitz, J. S., \& Tolin, D. F. (2006). Dimensionality of Somatic Complaints: Factor Structure and Psychometric Properties of the Self-Rating Anxiety Scale. Journal of Anxiety Disorders, 20, 543-561. https://doi.org/10.1016/j.janxdis.2005.08.002

Tabachnick, B. G., \& Fidell, L. S. (2001). Using Multivariate Statistics (4th ed.). Boston, MA: Allyn \& Bacon.

Vinothkumar, M., Arathi, A., Joseph, M., Nayana, P., Jishma, E. J., \& Sahana, U. (2016). Coping, Perceived Stress, and Job Satisfaction among Medical Interns: The Mediating Effect of Mindfulness. Industrial Psychiatry Journal, 25, 195-201. https://doi.org/10.4103/ipj.ipj_98_14

Wang, C., Cheng, Z., Yue, X. G., \& McAleer, M. (2020). Risk Management of COVID-19 by Universities in China. Journal of Risk and Financial Management, 13, 36. https://doi.org/10.3390/jrfm13020036

Wang, C., Pan, R., Wan, X., Tan, Y., Xu, L., Ho, C. S., \& Ho, R. C. (2020). Immediate Psychological Responses and Associated Factors during the Initial Stage of the 2019 Coronavirus Disease (COVID-19) Epidemic among the General Population in China. International Journal of Environmental Research and Public Health, 17, 1729. https://doi.org/10.3390/ijerph17051729

Wang, G., Zhang, Y., Zhao, J., Zhang, J., \& Jiang, F. (2020). Mitigate the Effects of Home Confinement on Children during the COVID-19 Outbreak. The Lancet, 395, 945-947. https://doi.org/10.1016/S0140-6736(20)30547-X

Wilder-Smith, A., \& Freedman, D. O. (2020). Isolation, Quarantine, Social Distancing and Community Containment: Pivotal Role for Old-Style Public Health Measures in the Novel Coronavirus (2019-nCoV) Outbreak. Journal of Travel Medicine, 27, taaa020. https://doi.org/10.1093/jtm/taaa020

World Health Organization (2020). Novel Coronavirus (2019-nCoV) Situation Report-1. https://www.who.int/docs/default-source/coronaviruse/situation-reports/20200121-sitr ep-1-2019-ncov.pdf

Worldometer (2020). COVID-19 Coronavirus Outbreak. https://www.worldometers.info/coronavirus/\#countries 
Wu, S., Xu, Z., Zhang, Y., \& Liu, X. (2019). Relationship among Psychological Capital, Coping Style and Anxiety of Chinese College Students. Rivista di psichiatria, 54, 264-268.

Xiao, H., Zhang, Y., Kong, D., Li, S., \& Yang, N. (2020a). The Effects of Social Support on Sleep Quality of Medical Staff Treating Patients with Coronavirus Disease 2019 (COVID-19) in January and February 2020 in China. Medical Science Monitor, 26, e923549. https://doi.org/10.12659/MSM.923921

Xiao, H., Zhang, Y., Kong, D., Li, S., \& Yang, N. (2020b). Social Capital and Sleep Quality in Individuals Who Self-Isolated for 14 Days during the Coronavirus Disease 2019 (COVID-19) Outbreak in January 2020 in China. Medical Science Monitor, 26, e923921. https://doi.org/10.12659/MSM.923921

Xiao, S. Y. \& Yang, D. S. (1987). The Impact of Social Support on Physical and Mental Health. Chinese Mental Health Journal, 1, 183-187.

Xiao, Y., \& Torok, M. E. (2020). Taking the Right Measures to Control COVID-19. The Lancet Infectious Diseases, 20, 523-524. https://doi.org/10.1016/S1473-3099(20)30152-3

Yang, Y., Li, W., Zhang, Q., Zhang, L., Cheung, T., \& Xiang, Y. T. (2020). Mental Health Services for Older Adults in China during the COVID-19 Outbreak. The Lancet Psychiatry, 7, e19. https://doi.org/10.1016/S2215-0366(20)30079-1

Yue, X. G., Shao, X. F., Li, R., Crabbe, M., Mi, L., Hu, S. et al. (2020). Risk Management Analysis for Novel Coronavirus in Wuhan, China. Journal of Risk and Financial Management, 13, 22. https://doi.org/10.3390/jrfm13020022

Zandifar, A., \& Badrfam, R. (2020). Iranian Mental Health during the COVID-19 Epidemic. Asian Journal of Psychiatry, 51, Article ID: 101990.

https://doi.org/10.1016/j.ajp.2020.101990

Zhai, Y., \& Du, X. (2020). Mental Health Care for International Chinese Students Affected by the COVID-19 Outbreak. The Lancet Psychiatry, 7, e22. https://doi.org/10.1016/S2215-0366(20)30089-4

Zhou, X. (2020). Psychological Crisis Interventions in Sichuan Province during the 2019 Novel Coronavirus Outbreak. Psychiatry Research, 286, Article ID: 112895. https://doi.org/10.1016/j.psychres.2020.112895 